\title{
Joule heating in ferromagnetic nanostripes with a notch
}

\author{
Eduardo Ramos, ${ }^{1}$ Cristina López, ${ }^{1}$ Johanna Akerman, ${ }^{1}$ Manuel Muñoz, ${ }^{2}$ and José L. Prieto ${ }^{1}$ \\ ${ }^{1}$ Instituto de Sistemas Optoelectrónicos y Microtecnología (ISOM), Universidad Politécnica de Madrid, \\ Avda. Complutense s/n, E-28040 Madrid, Spain \\ ${ }^{2}$ IMM-Instituto de Microelectrónica de Madrid (CNM-CSIC), Isaac Newton 8, PTM, E-28760 Tres Cantos, Madrid, Spain
}

(Received 20 February 2015; revised manuscript received 6 May 2015; published 2 June 2015)

\begin{abstract}
The temperature in a ferromagnetic nanostripe with a notch subject to Joule heating has been studied in detail. We first performed an experimental real-time calibration of the temperature versus time as a $100 \mathrm{~ns}$ current pulse was injected into a Permalloy nanostripe. This calibration was repeated for different pulse amplitudes and stripe dimensions and the set of experimental curves were fitted with a computer simulation using the Fourier thermal conduction equation. The best fit of these experimental curves was obtained by including the temperature-dependent behavior of the electrical resistivity of the Permalloy and of the thermal conductivity of the substrate $\left(\mathrm{SiO}_{2}\right)$. Notably, a nonzero interface thermal resistance between the metallic nanostripe and the substrate was also necessary to fit the experimental curves. We found this parameter pivotal to understand our results and the results from previous works. The higher current density in the notch, together with the interface thermal resistance, allows a considerable increase of the temperature in the notch, creating a large horizontal thermal gradient. This gradient, together with the high temperature in the notch and the larger current density close to the edges of the notch, can be very influential in experiments studying the current assisted domain wall motion.
\end{abstract}

DOI: 10.1103/PhysRevB.91.214404

PACS number(s): 81.07.Gf, 85.75.Bb, 44.05.+e, 75.50.Bb

\section{INTRODUCTION}

The possibility of manipulating the magnetization with spin polarized currents [1] in ferromagnetic nanostructures, together with the proposal of the racetrack memory [2], has encouraged intense research on the movement of magnetic domain walls (DWs) in ferromagnetic nanostripes. The racetrack memory relies on the precise movement of a sequence of magnetic bits (domains separated by DWs) in a memory element, which is a ferromagnetic nanostripe. The movement of this sequence of magnetic bits should be done exclusively by an electric current, via spin transfer torque. The minimum current required to move a DW in a nanostripe (critical current) is usually quite large, particularly in materials with in-plane anisotropy (such as Permalloy) or in nanostripes with engineered constrictions (notch), often used to pin reliably the DW at a particular position $[3,4,5]$. In these devices, current densities higher than $10^{12} \mathrm{~A} / \mathrm{m}^{2}$ are commonly required to achieve the depinning and the movement of the DW.

Understandably, the Joule heating generated by such a large current has been a matter of concern for researchers working in this field, even if the current is applied in short nanosecond pulses. A high temperature induced by Joule heating could bring the ferromagnetic material close to or even above its Curie temperature, which, in the case of a racetrack memory, could easily result in data corruption. Also, in most of the experiments of current assisted DW depinning in ferromagnetic nanostripes with a notch, the electric current generates important thermal gradients close to the contact pads [6] and also close to the notch, as we will show in this work. These thermal gradients could also affect the movement of the DW [7,8].

There have been only few experimental attempts to estimate the average temperature in magnetic nanostripes when an electric current flows through the device, using DC current [9], long current pulses $[10,11]$ or short nanosecond pulses [3,12]. The estimated temperature in these works is quite different for similar current densities although, as we will see in the conclusions, not necessarily contradictory. There are theoretical expressions available describing either an ideal two-dimensional (2D) geometry [13] or also a more realistic 3D geometry [14], although in this case (Ref. [14]), with the limitation of needing an experimental input to provide a value of temperature. Moreover, these expressions cannot provide the vital information of the local temperature at every point of the device when the nanostripe is not uniform (for instance, it has a notch) or when it has contact pads dissipating heat.

The local temperature at every point of a nanostripe device can only be obtained with a computer simulation and using the correct material properties extracted from an experimental calibration. Numerical methods have been applied recently to some particular problems of a magnetic nanostripe subject to Joule heating $[6,15]$. Despite the useful results in these works there are still aspects that need to be characterized in more detail. The material properties are usually considered constant with temperature and some of them change substantially as the nanostripe gets hotter. The current density is often considered constant, but it changes largely in a matter of nanoseconds when the stripe heats up, even if a current source is used, as this source may not respond fast enough to the change of resistance. Finally and notably, the interface between the metallic nanostripe and the substrate constitutes a thermal resistance although, to the best of our knowledge, it has been invariably neglected so far. The interface thermal resistance is a very influential parameter and it is required to fit the experimental results at different current densities, as we will see in the following sections.

In this work, using an experimental calibration and simulations with the software COMSOL [16], we evaluate the temperature in a Permalloy nanostripe with contact pads and 
a notch in the middle (one of the most popular geometries used so far), for different excitation conditions and using different substrates. The temperatures obtained inside the notch are higher than expected due to the interface thermal resistance and, consequently, large in-plane thermal gradients build up around the notch. These thermal gradients, together with the asymmetric current distribution inside the notch should be taken into account when analyzing experiments of current-assisted DW depinning.

\section{EXPERIMENTAL DETAILS}

\section{A. Experimental calibration of the temperature}

In order to select the correct values for the material properties required in the thermal simulation, we have performed experimental calibrations of resistance versus temperature $(R-T)$ and resistance versus current density $(R-J)$ in different Py nanostripes. The test samples were deposited by DC sputtering with the structure $\mathrm{Ta}(2) / \mathrm{Py}(10) / \mathrm{Ta}(2) / \mathrm{Pt}(1)$ on a $\mathrm{SiO}_{2}(400) / \mathrm{Si}$ substrate, where the numbers indicate the thickness in nanometers. The stripes were fabricated by liftoff, $12 \mu \mathrm{m}$ long and with widths ranging from $300 \mathrm{~nm}$ to $1 \mu \mathrm{m}$. The nanostripes are long enough to ignore the lateral cooling induced by the contact pads in the calibration $R-J$. We used stripes of different widths in order to test that the resistivity obtained in the calibration $R-T$ was similar in all of them and in order to infer the interface thermal resistance between the metallic stripe and the substrate.

First we performed the $R-T$ calibration on a cryostat from $200 \mathrm{~K}$ to the maximum temperature possible in our system, $500 \mathrm{~K}$. Each nanostripe was characterized by monitoring the resistance $R$ while the temperature was slowly increased from minimum to maximum. Then $R$ was also monitored while decreasing the temperature to check that the slope $R-T$ was the same and the stripe did not suffer any permanent damage. From these measurements we calculated the resistivity $\rho$ of the whole metallic stripe with the Py and the Ta and Pt layers. The width and the thickness of the stripes were accurately measured in each case with an atomic force microscope. The resistivity obtained was $\rho(T)=0.092 T+34.8$ in units of $\mu \Omega \mathrm{cm}$. This fit was very similar for nanostripes of different widths and it is also virtually the same as the experimental temperaturedependent resistivity used in Ref. [14].

Once the curve $R(T)$ was known for each wire, we proceeded with the real-time calibration of the resistance versus the current density $(R-J)$. The setup is shown in Fig. 1(a). A nanosecond pulse generator with output impedance $Z_{0}=50 \Omega$ was connected to the nanostripe with an impedance matched coplanar waveguide. The other end of the stripe was connected to a high-frequency oscilloscope with input impedance $Z_{0}=$ $50 \Omega$.

When a nanosecond pulse of amplitude $V_{p}$ is requested to the pulse generator, the current flowing through the stripe can be determined by the signal transmitted to the high-frequency oscilloscope. Figure 1(b) shows a $2.5 \mathrm{~V}$ and $100 \mathrm{~ns}$ pulse transmitted through a $500 \mathrm{~nm}$ wide nanostripe, as it is received in the oscilloscope. There is a clear decay in the amplitude of the pulse with time due to Joule heating. As the temperature rises, the resistance of the nanostripe increases (a)
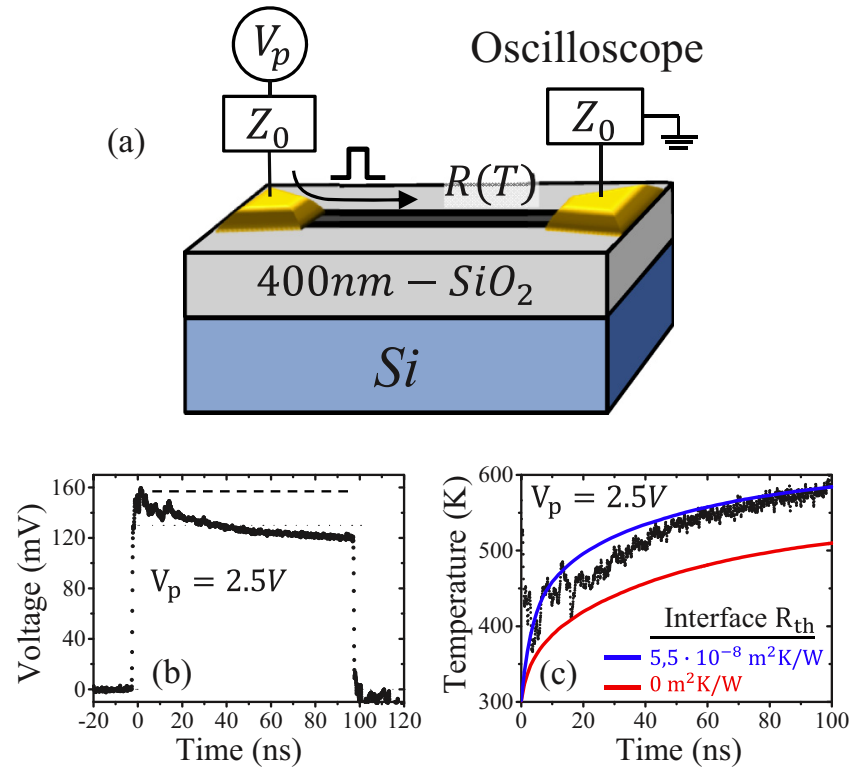

FIG. 1. (Color online) (a) Experimental setup for the real time measurement of the nanostripe resistance versus current density, using a $100 \mathrm{~ns}$ pulse. (b) Pulse of $V_{p}=2.5 \mathrm{~V}$ transmitted to the oscilloscope (current density of $\sim 0.4 \times 10^{12} \mathrm{~A} / \mathrm{m}^{2}$ at the end of the pulse). The decay in the amplitude reflects the increase in resistance of the nanostripe due to Joule heating. (c) Temperature versus time, obtained from the data in (b), formula (1) and the experimental calibration of resistivity versus temperature. The red and blue curves are Comsol simulations using zero and nonzero interface thermal resistance (see text).

and the current transmitted is smaller. The current flowing through the nanostripe can be extracted from the following simple formula,

$$
I[T(t)]=\frac{2 V_{p}}{R_{n s}(T)+2 Z_{0}},
$$

where $R_{n s}(T)$ is the resistance of the nanostripe, which is temperature dependent. The current $I[T(t)]$ can be extracted from the signal displayed in the oscilloscope as $I=V_{\text {osc }} / Z_{0}$ and, with the previous calibrations $R-T$ and $R-J$, we can convert the signal in the oscilloscope [Fig. 1(b)] into a temperature versus time $T(t)$ curve, as shown in Fig. 1(c). This experimental $T(t)$ curve can be measured for different excitation conditions $V_{p}$ as shown in Fig. 2(a) and for different stripes. Reproducing all these experimental curves with the computer simulations cannot be done unless the material properties introduced into COMSOL are correct and they have the correct behavior with temperature.

\section{B. Details of the computer simulations}

The thermal simulations were done with COMSOL, using the heat diffusion equation:

$$
\nabla^{2} T+\frac{Q}{k}=\frac{C_{V}}{k} \frac{\partial T}{\partial t}
$$

with $T$ the temperature, $k$ the thermal conductivity, $C_{V}$ the heat capacity per unit of volume and $Q$ the heating term, which for the case of Joule heating is given by $Q=\rho \cdot j^{2}$, with $\rho$ the 

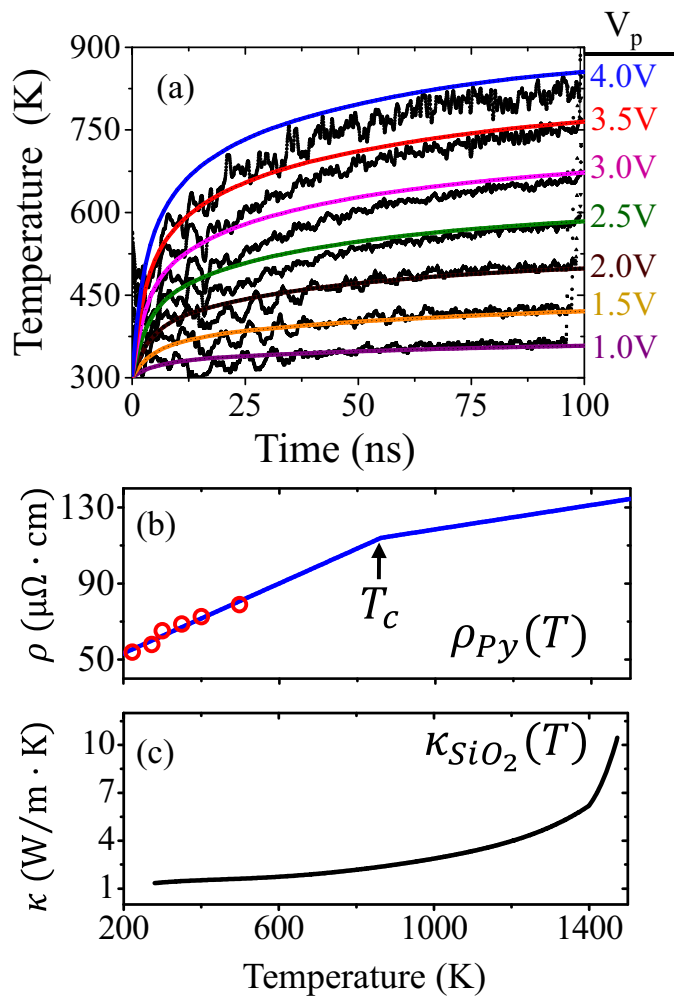

FIG. 2. (Color online) (a) Experimental temperature versus time curves for different amplitudes of pulse. As a guide, use the following conversion to extract the current density at the end of the $100 \mathrm{~ns}$ pulse $J=-0.018(V p)^{2}+0.186 V p+0.06$ in units of $10^{12} \mathrm{~A} / \mathrm{m}^{2}$. The color curves are the curves extracted from the simulations. The oscillations visible at small pulses are likely caused by the large resistance mismatch introduced by the nanostripes in the transmission line. Therefore they should not be interpreted as oscillations of the temperature. (b) Experimental calibration of the resistivity of the Permalloy nanostripes with temperature (red circles) and the curve extrapolated for higher temperatures from the data of Ref. [18], in blue. (c) Thermal conductivity of $\mathrm{SiO}_{2}$ with temperature. Data obtained from Comsol and Ref. [20].

electric resistivity of Py and $j$ the current density. This equation describes the transport of heat in a diffusive process and it is not necessarily applicable at the nanoscale. In our case, for a $\mathrm{SiO}_{2}$ substrate and for temperatures around or above its Debye temperature $(\sim 500 \mathrm{~K})$, the lifetime of phonons is in the range of $0.5 \mathrm{ps}$ and their mean-free path $1.5 \mathrm{~nm}$ or smaller [17]. These values are considerably shorter than the few nanoseconds of the current pulse used and smaller than the dimensions of the nanostripes and the cooling volume underneath. Therefore the system transports the heat in a diffusive regime and the use of the Fourier equation is justified.

In order to fit the experimental results, initially we used similar material parameters to the ones used by other authors. A good table with these material parameters can be found for instance in Refs. [6] and [15]. A single $T(t)$ curve for a particular voltage pulse, can be fitted easily by changing slightly the effective thickness of the nanostripe, as we do not know exactly how conductive the Ta layers are. On the other hand, the same effective thickness would not fit other $T(t)$ curves, especially at higher temperatures. We were forced therefore to consider the temperature dependency of the material properties, most of which are available in the internal database of COMSOL. We found three material properties of particular importance.

First, the resistivity with temperature for our Py was extrapolated for temperatures above its Curie temperature. The slope of $\rho(T)$ in ferromagnetic materials changes as the temperature crosses the Curie temperature [18,19]. Extrapolating our experimental data from $500 \mathrm{~K}$ to temperatures above the bulk Curie point of Py $(860 \mathrm{~K})$ is rather crude, but we found it necessary to fit all our experimental curves. Below Curie temperature we used $\rho(T)=0.092 T+34.8$ and above $\rho(T)=0.032 T+86.8$ in units of $\mu \Omega \mathrm{cm}$, extrapolating with the data provided in Ref. [18].

Second, the thermal conductivity of $\mathrm{SiO}_{2}$ can increase significantly for high temperatures due to photon conduction [20] and its behavior is displayed in Fig. 2(c). This is also important for high-voltage pulses, especially for the simulations performed in Sec. III, where the notch can reach very high temperatures.

Finally, we had to include a thermal resistance at the interface between the metallic nanostripe and the $\mathrm{SiO}_{2}$ substrate. The interface thermal resistance has been neglected in previous studies, although it is usually present at the interface between different materials and especially at the interface between metals and thermal insulators [21]. The value of the interface thermal resistance that fits best our experimental calibration curves is $5.5 \times 10^{-8} \mathrm{~m}^{2} \mathrm{~K} / \mathrm{W}$. This value is quite similar to the one found for other metals deposited on oxidized $\mathrm{Si}$ [22] and, as shown in Fig. 1(c), it can affect the results of the simulations to a large extent.

Using the temperature-dependent material properties and the interface thermal resistance we can fit all the experimental curves [see Fig. 2(a) for a $500 \mathrm{~nm}$ wide nanostripe]. The effective thickness of the metallic nanostripe that allowed the best fit was $12 \mathrm{~nm}$. This means that either some of the $\mathrm{Ta}$ in contact with the $\mathrm{SiO}_{2}$ substrate is oxidized and/or that the Ta became very resistive as a thin layer [23]. Figure 2(a) shows small deviations between simulations and experiments for the largest voltage pulses. We believe this is caused by the assumption of having an interface thermal resistance constant with temperature, when in reality it should also be temperature dependent [21]. The experimental determination of this dependency is beyond the scope of this work. Figure 2(a) shows also a larger deviation between simulations and experiments during the first nanoseconds of the pulse. This could be caused by the large impedance mismatch introduced by the nanostripe or by assuming a thermal interface resistance constant with temperature. In any case, the valuable information for this study is the temperature towards the end of the pulse.

We also checked that convection is totally negligible even for large values of the convection coefficient, corresponding to a very efficient forced convection. This is expected due to the nanometric scale of the problem.

\section{SIMULATION RESULTS}

Armed with the material properties extracted from fitting the experimental calibration curves in the previous section, we can now simulate the thermal behavior in nanostripes with 

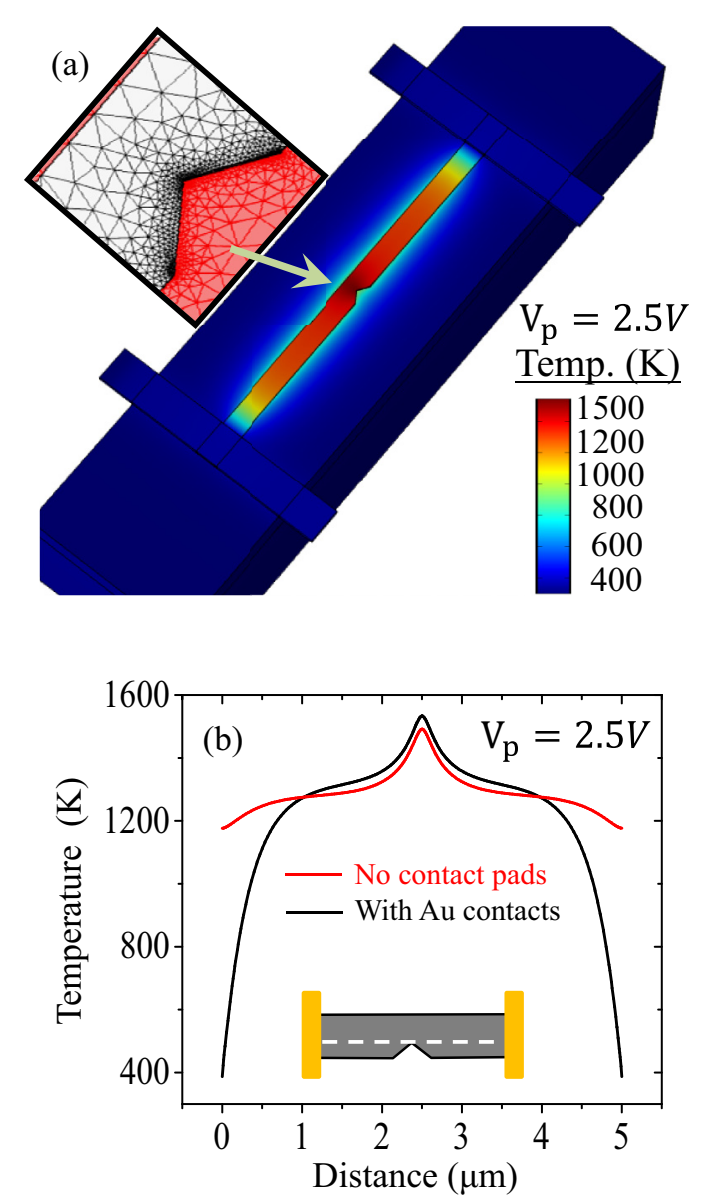

FIG. 3. (Color online) (a) Simulated nanostripe device with the temperature in the different sections represented by a color scale, at the end of a $100 \mathrm{~ns}$ and $2.5 \mathrm{~V}$ pulse. The inset shows the fine mesh around the notch. (b) Temperature profile along the length of the stripe by the white dotted line shown the cartoon. The red curve is for a stripe with no contact pads and the black curve for a stripe with Au contact Pads.

contact pads and with a notch in the middle. Figure 3(a) shows the typical geometry of a nanostripe used repeatedly in previous works exploring spin transfer assisted DW depinning. The contact pads are $500 \times 50 \mathrm{~nm}^{2}$ made of Au and they are in contact with the Py nanostripe and with the substrate next to the nanostripe. The nanostripe is $5 \mu \mathrm{m}$ long and $300 \times 12 \mathrm{~nm}^{2}$ and we have used the resistivity and effective thickness calculated in previous sections. The substrate is $\mathrm{SiO}_{2}(400) / \mathrm{Si}$ and it is simulated on a box of $4 \times 10 \times 20 \mathrm{~mm}$, although the outside planes are considered semi-infinite ("Infinite Element" option in the software). The nanostripe has a triangular notch in the middle $300 \mathrm{~nm}$ wide and $100 \mathrm{~nm}$ deep. We have used a very fine mesh around the notch to capture the steep gradients in temperature and current density [see inset to Fig. 3(a)].

Figure 3(b) shows the temperature profile in the nanostripe at the end of a $100 \mathrm{~ns}$ voltage pulse of $2.5 \mathrm{~V}$, with and without contact pads, along a line that touches the edge of the triangular notch [see Fig. 3(b)]. The average current density is almost constant after $100 \mathrm{~ns}$, as we will see later, and its value for a pulse of $2.5 \mathrm{~V}$ is $\sim 0.65 \times 10^{12} \mathrm{~A} / \mathrm{m}^{2}$. Note that this current density is larger than the one reported for the same $2.5 \mathrm{~V}$ pulse

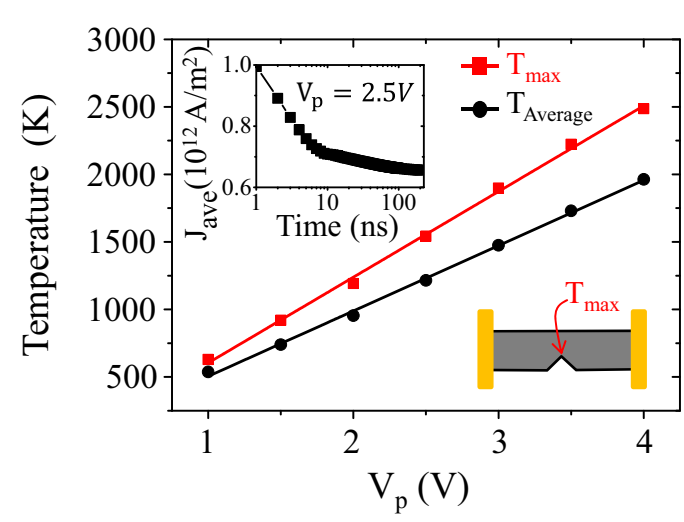

FIG. 4. (Color online) Average temperature in the nanostripe for different amplitudes of a $100 \mathrm{~ns}$ pulse (black circles) and maximum temperature reached at the tip of the triangular notch (red squares). The inset shows the behavior of the average current density flowing through the stripe with time, as the temperature and the resistance of the nanostripe increase.

in the calibration stripes (Figs. 1 and 2), as we are using now a shorter $5 \mu \mathrm{m}$ long stripe, which means less resistance and more current delivered by the pulse generator. As expected, the $\mathrm{Au}$ contact pads act as thermal sinks and they induce a steep thermal horizontal gradient, extending for about $1 \mu \mathrm{m}$ from the $\mathrm{Au}$ pad. The temperature reached in the nanostripe exceeds $1200 \mathrm{~K}$ and it goes to almost $1600 \mathrm{~K}$ at the tip of the notch. The reason behind such a high temperature is the poor thermal conductivity of the thick $\mathrm{SiO}_{2}$ layer. Unexpectedly, having contact pads induces a higher temperature in the center of the notch. The lower average temperature due to the contact pads leads to a smaller resistance of the nanostripe and as a consequence, to a higher current density delivered by the pulse generator.

The average temperature of the nanostripe can potentially be measured experimentally [3]. On the other hand, the maximum temperature of the notch is not accessible experimentally but very likely triggers the degradation or destruction of the device. Therefore it is important to have an idea of the temperature that the notch may have for a given voltage pulse or for a given average temperature. This information is provided in Fig. 4 for a triangular notch. In order to put these results in an experimental context, in our experience, a nanostripe with the dimensions studied would likely be destroyed for pulses of the order of $2.5 \mathrm{~V}$. For this voltage, the notch reaches a temperature higher than $1500 \mathrm{~K}$, close to the bulk melting point of Permalloy $(\sim 1700 \mathrm{~K})$, which may explain why the nanostripes break around that voltage of the pulse. The nanostripe would likely survive a single pulse larger than $2.5 \mathrm{~V}$, especially if it is only few nanoseconds long but, for such a large current density, the repeatability of the experiment should be always checked. This is again for a nanostripe deposited on a very thick $\mathrm{SiO}_{2}$ layer. For a thinner $\mathrm{SiO}_{2}$ layer or a more thermally conductive substrates, higher excitation pulses may be possible as we will see later.

The inset to Fig. 4 shows the behavior of the average current density with time. As the temperature of the nanostripe increases in the first nanoseconds of the pulse, the resistance increases and it causes a quick reduction of the current density, 

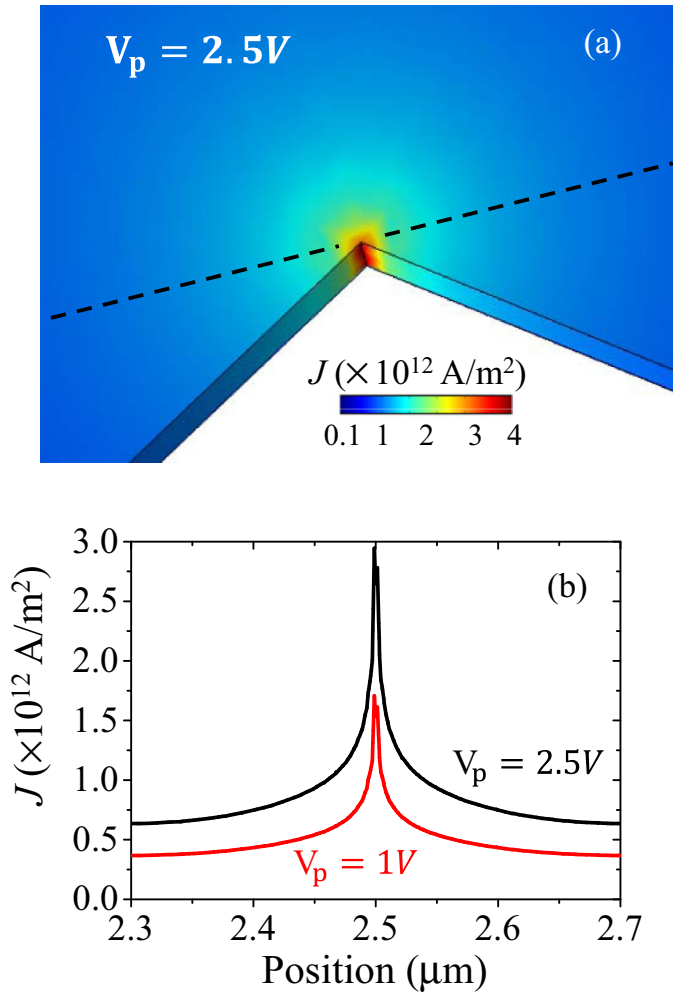

FIG. 5. (Color online) (a) Color-coded map of the current density distribution around the tip of the notch. (b) Current density along the black dotted line in (a) for two amplitudes of the pulse.

reaching an almost constant value after $100 \mathrm{~ns}$. This behavior is virtually the same (only displaced vertically) for different voltage pulses. The current density after $100 \mathrm{~ns}$ of a pulse of amplitude $V_{p}$ follows the expression $J_{\text {ave }}(t>100 \mathrm{~ns})=$ $0.17 \times V_{p}+0.24$ in units of $10^{12} \mathrm{~A} / \mathrm{m}^{2}$, for pulses between $1 \mathrm{~V}$ and $4 \mathrm{~V}$. This equation is specific for this particular nanostripe and substrate but it can be useful to compare our results with previous experimental estimations of the temperature in nanostripes subject to Joule heating (see Sec. IV).

As we have seen, if the substrate is not a good thermal conductor or if there is an interface thermal resistance, there is a substantial increase of the temperature in the notch as shown in Figs. 3 and 4. The current density increases in the constriction and it is particularly large at the tip of the notch as shown in Fig. 5. For a perfectly shaped triangular notch, the current density at the tip can be considerably larger than in the rest of the stripe. This effect is not only an issue from the thermal point of view, but also from the point of view of estimating the correct contribution of the spin transfer torque in DW depinning experiments. If a DW is pinned at the notch, the magnetization close to the tip of the notch is subject to more current and spin transfer than the rest of the DW.

The shape of the notch can improve the situation marginally. Figure 6 shows the current distribution and the temperature at the edge of a square notch and a circular notch. The square notch has been often used in previous works due to its high-efficiency pinning the DW [24]. As we can see in Figs. 6(a) and 6(b) though, a very large current density builds
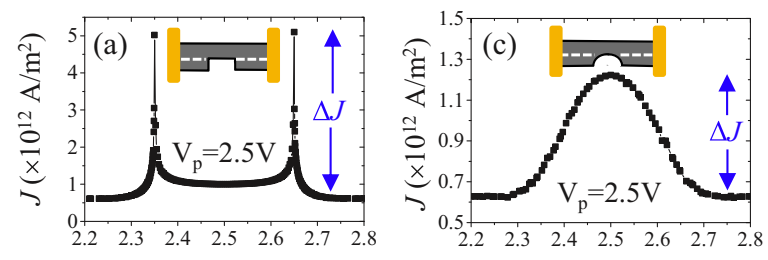

Distance $(\mu \mathrm{m})$
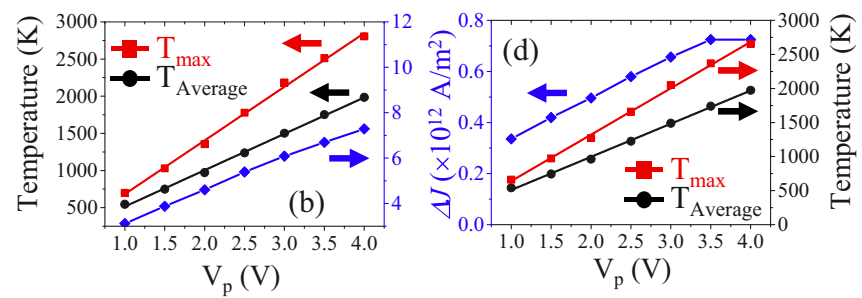

FIG. 6. (Color online) (a) Profile of the current density along the length of a nanostripe with a square notch, by the white dotted line shown in the cartoon. (b) Average temperature of the nanostripe (black circles) and maximum temperature at the edges of the notch (red circles) for various amplitudes of the pulse, with scale in the left $Y$ axis. The difference between the maximum current density and the current density in the stripe away from the notch $\Delta J$ is represented against the right $Y$ axis (blue rhomboids). (c) and (d) are plots equivalent to (a) and (b) respectively, for a notch with a circular shape.

up at the sharp edges of the notch, almost an order of magnitude larger than the average current density. This increase implies a maximum temperature even larger than in the case of a triangular notch. In fact, in our experience, stripes with a square notch get destroyed even for lower voltage pulses than stripes with triangular notches. Removing the sharp edges of the notch can reduce the concentration of current density as shown in Fig. 6(c). Nevertheless, the temperature increase is still quite substantial as it depends mainly on the width of the constriction.

Although the asymmetry of the current density around the notch is unavoidable, the buildup of temperature around the defect should be smaller for substrates with better thermal conductivity. This is analyzed in Fig. 7, where the current profile that is shown in Fig. 3(b), is recalculated for different thicknesses of the $\mathrm{SiO}_{2}$ layer, including zero thickness (i.e., pure $\mathrm{Si}$ substrate). Although there is a significant decrease of the average temperature as the thickness of the $\mathrm{SiO}_{2}$ is reduced, the temperature in the notch remains about $200 \mathrm{~K}$ higher than the rest of the stripe, independent of the substrate conductivity. This is intuitively unexpected. With Py being a metal (therefore a good thermal conductor), if the substrate is a fairly good thermal conductor as well, such as pure $\mathrm{Si}$ (purple curve, right triangle in Fig. 7), one would not expect a significant lateral buildup of temperature around the notch. This was indeed one of the results of Fangohr et al. [15]. In Fig. 4 of their work, these authors show the simulation for a Py nanostripe $5 \mu \mathrm{m} \times 150 \mathrm{~nm} \times 30 \mathrm{~nm}$, on a Si and a SiN substrate and with a triangular notch $45 \mathrm{~nm}$ deep in the middle. They found that, for a constant current density of $10^{12} \mathrm{~A} / \mathrm{m}^{2}$, the temperature at the notch was only a few degrees hotter than the rest of the stripe. We instead calculate a difference of about $100 \mathrm{~K}$ for a Si substrate (purple curve with a right triangle in 


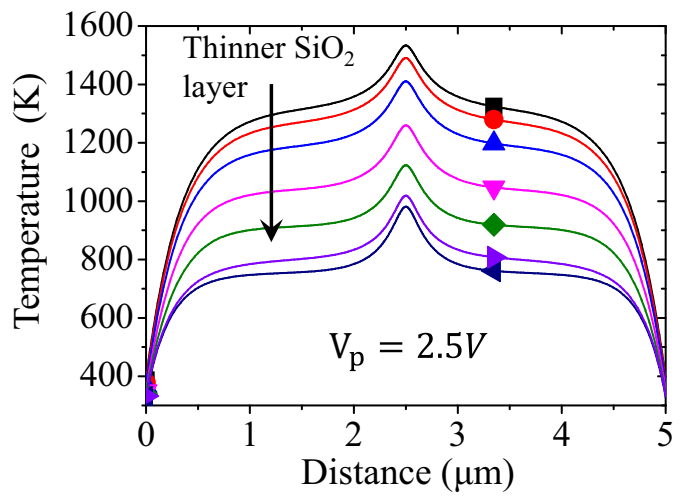

FIG. 7. (Color online) Temperature profile along the length of the nanostripe by the tip of the triangular notch as it was done in Fig. 2, for different thicknesses of the $\mathrm{SiO}_{2}$ layer: black square, $400 \mathrm{~nm}$; red circle, $300 \mathrm{~nm}$; top blue triangle, $200 \mathrm{~nm}$; bottom pink triangle, $100 \mathrm{~nm}$; green rhomboid, $50 \mathrm{~nm}$; right purple triangle for pure $\mathrm{Si}$ substrate; and left dark blue triangle for pure Sapphire substrate. The thermal resistance of the $\mathrm{SiO}_{2} / \mathrm{Si}$ interface is in the range of $10^{-9} \mathrm{~m}^{2} \mathrm{~K} / \mathrm{W}$, so it should have little influence in the final result.

Fig. 7). The main reason for this difference is that Fangohr et al. assumed perfect thermal contact between the stripe and the substrate while we needed to include an interface thermal resistance to fit our calibration $T(t)$ curves. This highlights the importance of using a correct value for the interface thermal resistance in order to predict the temperature at every point of the nanostripe.

\section{CONCLUSIONS}

Before discussing our results in light of previous experimental works, we draw the attention to the heating term $Q=\rho \cdot j^{2}$ in Eq. (2). It represents the heat generated per unit of volume and it should have the same value in experiments using the same current density and the same resistivity of the metallic nanostripe. It is important to note then that the total heat generated for a constant $\rho$ and $j$ increases with the cross section of the nanostripe. For instance, a nanostripe of cross-section $300 \times 10 \mathrm{~nm}^{2}$ would generate five times more heat per unit of length than a stripe with cross-section $120 \times 5 \mathrm{~nm}^{2}$, with only about twice the area to dissipate it.

Yamaguchi et al. [10,11], using long $5 \mu$ s current pulses (which can be assumed DC as the steady state is almost reached after only $100 \mathrm{~ns}$ ) estimated a temperature increase of $830 \mathrm{~K}$ in a $240 \times 10 \mathrm{~nm}^{2}$ Py nanostripe deposited on a $\mathrm{SiO}_{2}(100 \mathrm{~nm}) / \mathrm{Si}$ substrate for a current density of $7.5 \times$ $10^{11} \mathrm{~A} / \mathrm{m}^{2}$. This temperature is sufficiently high to bring the Py over its Curie temperature as their MFM images suggested. Although we do not have information of the buffer material used in their work, we estimate a very similar temperature for that current density in steady state, using the resistivity and the interface thermal resistance of our stripes. Yamaguchi et al. fitted their experimental results to the 2D model described in Ref. [13], but they needed a correction factor to raise the predicted temperature to the experimental values. The interface thermal resistance may be the origin of this correction factor.
Vernier et al. [9], using DC current and resistive measurements, estimated a mild temperature increase of $100 \mathrm{~K}$ for a current density of $6 \times 10^{11} \mathrm{~A} / \mathrm{m}^{2}$ flowing in a $120 \times 5 \mathrm{~nm}^{2}$ Py stripe deposited on a $\mathrm{SiO}_{2}(600 \mathrm{~nm}) / \mathrm{Si}$ substrate. This is apparently a low temperature for that current density looking to our results and the results of Yamaguchi et al. [10,11]. As explained at the beginning of this section, the stripe used by Vernier et al. had a cross-section five times smaller than the one used by us or by Yamaguchi. This implies, assuming similar material properties, five times less heat generated for the same current density, which can easily justify the $400 \mathrm{~K}$ measured by Vernier. In fact, for larger $500 \times 300 \mathrm{~nm}^{2}$ Py stripes (a cross-section five times larger than ours) deposited on a SiN substrate, Towaga et al. [19] reached the Curie temperature with only $2 \times 10^{11} \mathrm{~A} / \mathrm{m}^{2}$. This result reinforces the importance of looking at the cross section of the nanostripe in order to do a rough comparison of different experimental results, even if the material properties are considered the same. In any case, the resistivity of the Py nanostripe can change significantly depending on the deposition method and conditions $[25,26]$. Therefore, a comparison at a glance between different experimental results can be very challenging.

Hayashi [3,12] dealt with the problem of estimating experimentally the dynamic temperature in Py nanostripes as a short (tens of nanoseconds) current pulse flows through the device. He used different widths and thicknesses of nanostripes deposited over a $\mathrm{SiO}_{x}(25 \mathrm{~nm}) / \mathrm{Si}$ substrate. These stripes were all $4 \mu \mathrm{m}$ long and they were contacted at both ends by contact pads. As we have seen, these pads act as a heat sink and reduce the average temperature of the nanostripe. The temperature measured at the end of a $100 \mathrm{~ns} 2.5 \mathrm{~V}$ pulse, for a $300 \times 10 \mathrm{~nm}^{2}$ thick stripe was about $600 \mathrm{~K}$. In Fig. 7, the plot for a $25 \mathrm{~nm}$ thick $\mathrm{SiO}_{2}$ would be between the green and the purple curve and our estimated average temperature would be about $800 \mathrm{~K}$. The nanostripes used in Ref. [3] were shorter than ours and their resistivity smaller, which means lower resistance and a higher current density for the same amplitude of the pulse. Therefore, the lower temperature reported in Ref. [3] can only be explained by a very low interface thermal resistance. With the material properties provided in Ref. [12], we estimated that the $\mathrm{Fe} / \mathrm{AlO}_{x}$ buffer they used induces a fairly small $1 \times 10^{-8} \mathrm{~m}^{2} \mathrm{~K} / \mathrm{W}$ interface thermal resistance.

We can see, therefore, that the sometimes considerably different experimental estimations of temperature in nanostripes due to Joule heating are not necessarily contradictory. The choice of substrate and the dimensions of the nanostripe play, of course, a pivotal role in the final temperature. There are also other very influential parameters that may not be easily controllable, such as the resistivity of the metallic nanostripe or the interface thermal resistance between the stripe and the substrate. In fact, in light of our results, successful studies of current-induced DW motion may only be possible with a very low interface thermal resistance and/or a very good thermally conductive substrate such as diamond. In particular, when the nanostripe has a notch and there is interface thermal resistance, the temperature inside the notch builds up significantly, providing a strong in-plane thermal gradient. Additionally, we have shown that small regions around the edges of the notch 
are subject to a much larger current density than the average current density in the nanostripe. This effect can also influence the probability of DW depinning as it may bring local areas of the nanostripe above the Curie temperature or enhance the spin transfer torque locally in the region where the DW is closer to the edges of the notch. All these considerations should be taken into account when evaluating the strength of the spin transfer torque in current-induced DW movement and/or depinning experiments.

\section{ACKNOWLEDGMENT}

This work was funded by the Ministerio de Ciencia e Innovación MAT2011-28532-C03-03 and MAT2009-08771.
[1] J. C. Slonczewski, J. Magn. Magn. Mater. 159, L1 (1996).

[2] S. S. P. Parkin, M. Hayashi, and L. Thomas, Science 320, 190 (2008).

[3] M. Hayashi, L. Thomas, C. Rettner, R. Moriya, X. Jiang, and S. S. P. Parkin, Phys. Rev. Lett. 97, 207205 (2006).

[4] M. Hayashi, L. Thomas, C. Rettner, R. Moriya, and S. S. P. Parkin, Nature Phys. 3, 21 (2007).

[5] J. Akerman, M. Muñoz, M. Maicas, and J. L. Prieto, J. Appl. Phys. 115, 183909 (2014).

[6] J. Torrejon, G. Malinowski, M. Pelloux, R. Weil, A. Thiaville, J. Curiale, D. Lacour, F. Montaigne, and M. Hehn, Phys. Rev. Lett. 109, 106601 (2012).

[7] K. Uchida, S. Takahashi, J. Ieda, K. Harii, K. Ikeda, W. Koshibae, S. Maekawa, and E. Saitoh, J. Appl. Phys. 105, 07C908 (2009).

[8] P. Möhrkea, J. Rhensius, J. U. Thiele, L. J. Heyderman, and M. Kläui, Solid State Commun. 150, 489 (2010).

[9] N. Vernier, D. A. Allwood, D. Atkinson, M. D. Cooke, and R. P. Cowburn, Europhys. Lett. 65, 526 (2004).

[10] A. Yamaguchi, S. Nasu, H. Tanigawa, T. Ono, K. Miyake, K. Mibu, and T. Shinjo, Appl. Phys. Lett. 86, 012511 (2005).

[11] A. Yamaguchi, A. Hirohata, T Ono, and H. Miyajima, J. Phys.: Condens. Matter 24, 024201 (2012).

[12] M. Hayashi, Ph.D. thesis, Stanford University, 2006.

[13] C.-Y. You, I.-M. Sung, and B.-K. Joe, Appl. Phys. Lett. 89, 222513 (2006).

[14] K.-J. Kim, J.-C. Lee, S.-B. Choe, and K.-H. Shin, Appl. Phys. Lett. 92, 192509 (2008).
[15] H. Fangohr, D. S. Chernyshenko, M. Franchin, T. Fischbacher, and G. Meier, Phys. Rev. B 84, 054437 (2011).

[16] COMSOL MULTIPHYSICS ${ }^{\circledR}$. 2011. Version 4.2a. COMSOL, Inc., Burlington, MA, USA.

[17] J. M. Larkin and A. J. H. McGaughey, Phys. Rev. B 89, 144303 (2014).

[18] C. Y. Ho, M. W. Ackerman, K. Y. Wu, T. N. Havill, R. H. Bogaard, R. A. Matula, S. G. Oh, and H. M. James, J. Phys. Chem. Ref. Data 12, 183 (1983).

[19] Y. Togawa, T. Kimura, K. Harada, T. Matsuda, A. Tonomura, and Y. Otani, Appl. Phys. Lett. 92, 012505 (2008).

[20] D. W. Lee, and W. d. Kingery, J. Am. Ceram. Soc. 43, 594 (1960).

[21] D. G. Cahill, W. K. Ford, K. E. Goodson, G. D. Mahan, A. Majumdar, H. J. Maris, R. Merlin, and S. R. Phillpot, J. Appl. Phys. 93, 793 (2003).

[22] T. Yamane, N. Nagai, S. Katayama, and M. Todoki, J. Appl. Phys. 91, 9772 (2002).

[23] K. Stella, D. Bürstel, S. Franzka, O. Posth, and D. Diesing, J. Phys. D: Appl. Phys. 42, 135417 (2009).

[24] J. Akerman, M. Muñoz, M. Maicas, and J. L. Prieto, Phys. Rev. B 82, 064426 (2010).

[25] G. Nahrwold, J. M. Scholtyssek, S. Motl-Ziegler, O. Albrecht, U. Merkt, and G. Meier, J. Appl. Phys. 108, 013907 (2010).

[26] G. Nahrwold, L. Bocklage, J. M. Scholtyssek, T. Masuyama, B. Krüger, U. Merkt, and G. Meier, J. Appl. Phys. 105, 07D511 (2009). 